\title{
Degradação luminosa e Retenção Foliar dos Corantes Azul BRILHANTE FDC-1 E AMARElo TARTRASina FDC-5 UTILIZAdos Como TRAÇADORES EM PULVERIZaÇões ${ }^{1}$
}

\author{
Luminous Degradation and Foliage Retention of Dyes Fd\&C Blue no. 1 and Fd\&C Yellow no. 5 \\ Used as Spray Tracers
}

\author{
MARCHI, S.R. ${ }^{2}$; MARTINS, D. ${ }^{3}$, COSTA, N.V. ${ }^{2}$; TERRA, M.A. ${ }^{2}$ e \\ NEGRISOLI, E. ${ }^{2}$
}

\begin{abstract}
RESUMO - Três estudos foram conduzidos no Núcleo de Pesquisas Avançadas em Matologia (NUPAM) pertencente à UNESP/FCA - campus de Botucatu-SP, com o objetivo de avaliar a estabilidade dos corantes Azul Brilhante FDC-1 e Amarelo Tartrasina FDC-5 quanto a diferentes períodos de exposição à luz solar e contato com folhas de Eichhornia crassipes. No primeiro estudo, soluções de 0,3125, 0,625, 1,25, 2,5, 5, 10 e 20 ppm dos corantes Azul Brilhante FDC-1 e Amarelo Tartrasina FDC-5 foram acondicionadas em tubos de quartzo hermeticamente fechados e submetidos a 0, 0,5, 1, 2, 4, 6 e 10 horas de exposição à luz solar e ao escuro. Ao final de cada período, amostras de $10 \mathrm{~mL}$ foram retiradas dos tubos e analisadas. No segundo estudo, os tratamentos foram dispostos no esquema fatorial $2 \times 7$ : duas condições luminosas (escuro e pleno sol) e sete períodos de exposição $(0,0,5,1,2,4$, 6 e 10 horas), com seis repetições. Com o auxílio de micropipeta, oito gotas de $5 \mu \mathrm{L}$ das soluções Azul Brilhante e Amarelo Tartrasina a 4.000 ppm foram depositadas em placas de Petri de vidro. Após o término dos períodos de exposição, as placas foram lavadas com $50 \mathrm{~mL}$ de água destilada, com o objetivo de extrair o corante depositado sobre elas. No terceiro estudo, adotaram-se os mesmos tratamentos do segundo experimento, com quatro repetições, porém as soluções foram depositadas sobre as folhas de plantas de Eichhornia crassipes. Foram adotados também os mesmos procedimentos de extração dos corantes após o término dos periodos de exposição. As soluções finais obtidas nos três estudos foram submetidas à leitura óptica de absorbância em espectrofotômetro UV-visível nos comprimentos de onda de 630 e $427 \mathrm{~nm}$, para os corantes Azul Brilhante FDC-1 e Amarelo Tartrasina FDC-5, respectivamente. As várias concentrações das soluções de ambos os corantes não sofreram degradação pela luz solar quando submetidas aos vários períodos de incidência luminosa nos tubos de quartzo (ambiente fechado), visto que as curvas de recuperação apresentaram equações semelhantes àquelas concentrações que foram mantidas no escuro. A mesma estabilidade também foi observada quando os corantes foram submetidos à luz solar em ambiente aberto, ou seja, nas placas de Petri. O corante Amarelo Tartrasina também se apresentou muito estável quando depositado sobre as folhas de E. crassipes, independentemente da exposição ou não à luz solar. Para o corante Azul Brilhante, ocorreram significativas perdas de 7,8 e 18,6\% quando esteve depositado na superficie da folha de aguapé pelo período de 10 horas sob condições de escuro e plena luz solar, respectivamente.
\end{abstract}

Palavras-chave: tecnologia de aplicação, estabilidade, adsorção foliar, aguapé, Eichhornia crassipes.

ABSTRACT - Three trials were carried out at Núcleo de Pesquisas Avançadas em Matologia (NUPAM), FCA/UNESP - Campus de Botucatu, SP, Brazil, to evaluate the stability of dyes FD\&C Blue no. 1 and FD\&C Yellow no. 5 when exposed to different periods of solar light and Eich hornia crassipes leaf contact. Solutions at 0,3125, 0,625, 1,25, 2,5, 5, 10 and 20 ppm of both dyes were prepared and conditioned into Quartz tubes hermetically closed and submitted to several

1 Recebido para publicação em 3.1.2005 e na forma revisada em 15.3.2005.

2 Eng.-Agr., discente de Pós-graduação, Dep. de Produção Vegetal-Agricultura, UNESP/FCA <marchi@fca.unesp.br>; ${ }^{3}$ Prof. Livre Docente., Dep. Produção Vegetal-Agricultura, UNESP/FCA, Caixa Postal 237, 18603-970 Botucatu-SP. 
solar light and darkness periods, as follows 0; 0,5; 1; 2; 4; 6 and 10 hours. Samples of $10 \mathrm{~mL}$ were collected from the tubes after the end of each period and left to posterior analysis. The second trial treatments were designed as a factorial 2x7: 2 light conditions (sunlight or darkness) and 7 exposure timings $(0 ; 0,5 ; 1 ; 2 ; 4 ; 6$ and 10 hours), with six replications. Eight drops of $5 \mu L$ of FD\&C Blue no. 1 and FD\&C Yellow no. 5 solutions at 4,000 ppm were placed on glass Petri dishes, using a micropipette. Petri dishes were washed with $50 \mathrm{~mL}$ of distilled water immediately after each exposure period ending to extract the dyes deposited on it. The same treatments were used in the third trial, with four replications. However, the coloring solutions were dropped on Eichhornia crassipes foliar surfaces. The same dye extraction procedures were also used after each exposure period ending. The final solutions obtained from the three trials were submitted to absorbance optical analysis at spectrophotometer UV-Visible on wave length of 630 and $427 \mathrm{~nm}$ for FD\&C Blue no. 1 and FD\&C Yellow no. 5 dyes, respectively. Both coloring solutions at different concentrations were not degraded by solar light after being submitted to each exposure period of sunlight incidence into the Quartz tubes (closed situation), since recovering curves showed practically the same equations when compared to the dye solutions kept under darkness conditions. The same stability was observed when the dye solutions were submitted to sun light incidence in open ambient, i.e., on Petri dishes. FD\&C Yellow no. 5 showed high stability when dropped over E. crassipes leaves, regardless of the light condition. However, statistical losses of $7.8 \%$ and $16.8 \%$ were observed when FD\&C Blue no. 1 was dropped over the surface of water hyacinth leaves during $10 \mathrm{~h}$ under darkness and sunlight incidence conditions, respectively.

Key words: spraying technology, stability, foliar adsorption, water hyacinth Eichhornia crassipes.

\section{INTRODUÇÃO}

A avaliação dos depósitos da calda aplicada em alvos naturais ou artificiais é o método mais representativo para entender diversos aspectos relacionados à pulverização de defensivos agrícolas. No entanto, os custos dessas pesquisas podem ser um fator limitante na condução dos estudos quando as determinações são realizadas utilizando-se os próprios defensivos agrícolas, visto que necessitam de reagentes apropriados, equipamentos sofisticados e de pessoas treinadas para a realização das análises (Matuo, 1988). Assim, o uso de traçadores é muito atrativo, em virtude da facilidade de sua visualização ou remoção das folhas ou alvos coletores diretamente pela utilização de água.

Por sua vez, as soluções traçadoras devem ser sensiveis à detecção; possibilitar o uso em análise quantitativa com rapidez; ser solúveis quando misturadas à calda, com efeitos físicos mínimos na pulverização e menor evaporação das gotas; ter propriedades distintas para se diferenciar de outras substâncias; e ser estáveis, atóxicas e de baixo custo (Yates \& Akesson, 1963). Os corantes alimentícios Azul Brilhante e Amarelo Tartrasina catalogados internacionalmente pela "Food, Drug \& Cosmetic" (FD\&C) como FD\&C Blue n. 1 e FD\&C Yellow n.5, respectivamente, atendem a todas as exigências requeridas de um traçador citadas anteriormente.

Os primeiros relatos da utilização dos dois corantes alimentícios como traçadores de calda de pulverização são recentes e datam da década de 1990. Hayden et al. (1990), com o objetivo de comparar duas condições de trabalho em alvo natural, utilizando uma mesma solução para determinar a quantidade depositada em cada teste, escolheram dois corantes alimentícios solúveis em água (FD\&C n. 1 e FD\&C n. 6) e concluíram que o método foi preciso e barato para as pesquisas de avaliações de depósitos em plantas. O corante FD\&C Yellow n. 5 foi utilizado por Pergher et al. (1997) para comparar a deposição de gotas de pulverização em folhas e as perdas de três diferentes turboatomizadores usados em viticultura cultivada em espaldeira.

No Brasil, os estudos referentes à estabilidade dos corantes, às características físicas das caldas e ao desenvolvimento da metodologia de análise quantitativa envolvendo os corantes FD\&C Blue n. 1 e FD\&C Yellow n. 5 foram inicialmente realizados por Palladini (2000). A partir desta data, a quase totalidade dos 
pesquisadores tem se utilizado desta técnica para estudar a quantidade de calda depositada pelas pulverizações realizadas em alvos naturais ou artificiais (Souza et al., 2000; Tofoli, 2001; Negrisoli et al., 2002; Costa et al., 2004; Silva et al., 2004; Terra et al., 2004).

No entanto, a maioria desses pesquisadores tem utilizado esta metodologia em plantas integras nos seus respectivos estudos de análise quantitativa dos depósitos de calda pulverizada e, geralmente, realizado as aplicações em campo, à plena luz do sol. Tal procedimento pode estar conduzindo à obtenção de resultados dúbios, uma vez que a metodologia descrita por Palladini (2000) foi desenvolvida em folhas destacadas e por períodos alternados de exposição à luz solar.

Nesse sentido, o presente trabalho teve por objetivo avaliar a estabilidade dos corantes FD\&C Blue n. 1 e FD\&C Yellow N. 5 em relação à degradação luminosa e absorção foliar quando depositados em folhas de plantas integras de Eichhornia crassipes e mantidos em longos períodos de contato foliar e exposição à luz solar.

\section{MATERIAL E MÉTODOS}

Três estudos foram conduzidos em junho de 2003 no Núcleo de Pesquisas Avançadas em Matologia (NUPAM), pertencente ao Departamento de Produção Vegetal/Agricultura da FCA/UNESP, campus de Botucatu-SP. A média da radiação solar no plano inclinado fixo (RIF) otimizado para Botucatu-SP, observada no período de condução dos ensaios, foi de $543,71 \mathrm{~W} \mathrm{~m}^{-2}$.

O primeiro estudo teve por finalidade analisar a estabilidade de diferentes concentrações dos corantes FD\&C Blue n. 1 e FD\&C Yellow n. 5, também denominados de Azul Brilhante e Amarelo Tartrasina, respectivamente, quando submetidas a diferentes períodos de exposição direta à luz solar em alvos artificiais. Soluções-estoque dos dois corantes a 0,$3125 ; 0,625 ; 1,25 ; 2,5 ; 5,0 ; 10,0$; e 20,0 ppm foram preparadas em água destilada e posteriormente fracionadas e acondicionadas em tubos de quartzo de $100 \mathrm{~mL}$ hermeticamente fechados, para evitar as perdas por evaporação. Os tubos de quartzo, além de forma arredondada, também possuem a propriedade de deixar passar $100 \%$ dos raios solares que atingem sua superficie.

As diferentes concentrações dos corantes Azul Brilhante e Amarelo Tartrasina foram dispostas em baterias e submetidas a duas condições de luminosidade: ausência total de luminosidade em câmara escura e exposição direta à luz solar, por períodos de 0 (testemunha); 0,5; 1,0;2,0;4,0;6,0; e 10,0 horas. A bateria de tubos mantida a plena luz solar foi inclinada para o Norte Verdadeiro da Terra em um ângulo inclinado correspondente à latitude de Botucatu-SP mais $10^{\circ}$, com o objetivo de otimizar a incidência da luz solar para o período de inverno (RIF). Ao final de cada período de exposição, amostras de $10 \mathrm{~mL}$ foram retiradas dos tubos, acondicionadas em frascos plásticos de cor preta e destinadas para posterior leitura de absorbância em espectrofotômetro.

No segundo estudo, os tratamentos foram dispostos no esquema fatorial $2 \times 7$, sendo duas condições de luminosidade (escuro e pleno sol) e sete períodos de exposição $(0 ; 0,5 ; 1,0$; 2,$0 ; 4,0 ; 6,0 ;$ e 10,0h), com seis repetições. Oito gotas de $5 \mu \mathrm{L}$ das soluções dos corantes Azul Brilhante e Amarelo Tartrasina a $4.000 \mathrm{ppm}$ em água destilada foram depositadas em placas de Petri de vidro, com auxílio de micropipetas. As placas de Petri permaneceram destampadas durante todo o periodo de condução do ensaio, visando avaliar possíveis perdas por evaporação ou volatilização dos corantes em alvos artificiais, uma vez que elas são confeccionadas de material inerte e permitem a total extração dos corantes. Após o término dos períodos de exposição, as placas foram lavadas com $50 \mathrm{~mL}$ de água destilada, e as novas soluções obtidas também foram acondicionadas em frascos plásticos de cor preta e destinadas para posterior leitura.

No terceiro estudo, foram adotados os mesmos tratamentos utilizados no segundo, com quatro repetições. Neste caso, as oito gotas de $5 \mu \mathrm{L}$ dos dois corantes foram depositadas sobre folhas integras de plantas de aguapé (Eichhornia crassipes) cultivadas em vasos contendo $100 \mathrm{~L}$ de água. Esse procedimento teve por objetivo verificar se ocorre algum tipo de reação entre os corantes e o alvo natural no caso, as folhas do aguapé. Adotaram-se

Planta Daninha, Viçosa-MG, v. 23, n. 2, p. 287-294, 2005 
também os mesmos procedimentos de extração e acondicionamento dos corantes após o término dos períodos de exposição.

Todas as amostras e soluções obtidas nos três estudos foram prontamente analisadas em espectrofotômetro UV-visível de duplo feixe, modelo Cintra 20, operando com 3,0 mm de caminho óptico, cujos resultados em absorbância nos comprimentos de onda de 630 e $427 \mathrm{~nm}$ para os corantes Azul Brilhante e Amarelo Tartrasina, respectivamente, foram transformados em ppm por meio da confecção de curvas-padrão a partir de concentrações conhecidas.

Os resultados de absorbância e ppm obtidos no primeiro estudo foram utilizados na confecção das linhas de tendência e suas respectivas equações para os corantes Azul brilhante e Amarelo Tartrasina, em cada uma das condições de luminosidade e período de exposição. Já os resultados obtidos em ppm no segundo e terceiro estudos foram submetidos à análise de variância pelo teste $\mathrm{F}$, sendo as médias dos tratamentos comparadas pelo teste t a $5 \%$ de probabilidade.

\section{RESULTADOS E DISCUSSÃO}

Na Figura 1 estão apresentadas as curvas de regressão linear e suas respectivas equações obtidas no primeiro estudo, a partir das leituras de absorbância realizadas nas amostras retiradas das soluções do corante Azul Brilhante mantidas hermeticamente fechadas nos tubos de quartzo durante os diferentes períodos de exposição ou não à luz solar. Podese notar que os coeficientes angulares das equações foram muito semelhantes, independentemente do período e da condição de luminosidade a que as soluções foram submetidas.

Os coeficientes angulares divergiram somente na terceira casa decimal após a vírgula; a maior diferença foi observada entre as soluções mantidas por $0,5 \mathrm{~h}$ a plena luz solar e no escuro. Mesmo assim, a aplicação da fórmula ao limite extremo de absorbância de $3,0 \mathrm{~nm}$, representado no gráfico, conduz os resultados a uma diferença de 0,0805 ppm para mais ou para menos na concentração da solução a ser calculada.

Planta Daninha, Viçosa-MG, v. 23, n. 2, p. 287-294, 2005
Resultados semelhantes foram observados para o corante Amarelo Tartrasina, uma vez que os coeficientes angulares das equações obtidas a partir das leituras de absorbância realizadas nas amostras também diferiram somente na terceira casa decimal após a vírgula (Figura 2). Curiosamente, a maior diferença entre os coeficientes angulares foi observada entre as amostras obtidas imediatamente após o seu preparo e sem antes mesmo de serem fracionadas e acondicionadas nos tubos de quartzo, ou seja, submetidas a $0 \mathrm{~h}$ de exposição ou não à luz solar. Neste caso, a diferença entre os coeficientes angulares foi de 0,006 ponto, o que poderia ocasionar uma diferença máxima de 0,0097 ppm para mais ou para menos na determinação da concentração final da solução, quando utilizado o limite máximo de $0,8 \mathrm{~nm}$ de absorbância representado no gráfico.

As pequenas diferenças observadas entre os coeficientes angulares das expressões obtidas em cada intervalo de exposição ou não à luz solar e as máximas diferenças calculadas através da aplicação das fórmulas de 0,0805 e 0,0097 ppm para os corantes Azul Brilhante e Amarelo Tartrasina podem ser consideradas como despreziveis e possivelmente decorrentes de erros experimentais não-controlados, visto que estes valores, em ppm, estão muito abaixo dos limites de sensibilidade para detecção em espectrofotômetro dos dois corantes, os quais são de 0,246 e 1,953 ppm, respectivamente (Palladini, 2000). Frick \& Huck (1995) comentam que os corantes Azul Brilhante e Amarelo Tartrasina, quando utilizados na indústria alimentícia, apresentam, respectivamente, baixa e alta instabilidades à luz. Entretanto, os autores não relatam os períodos mínimos em que os corantes podem ser submetidos à condição de incidência luminosa intensa sem que ocorram perdas consideráveis dos corantes. Neste primeiro estudo, os resultados sugerem que os corantes Azul Brilhante e Amarelo Tartrasina não apresentam degradação luminosa quando expostos diretamente à luz solar por um periodo de até 10,0 horas.

No segundo estudo, foi possivel verificar que ambos os corantes não apresentaram nenhuma variação quando depositados diretamente nas placas de Petri e submetidos aos 


\section{Escuro}
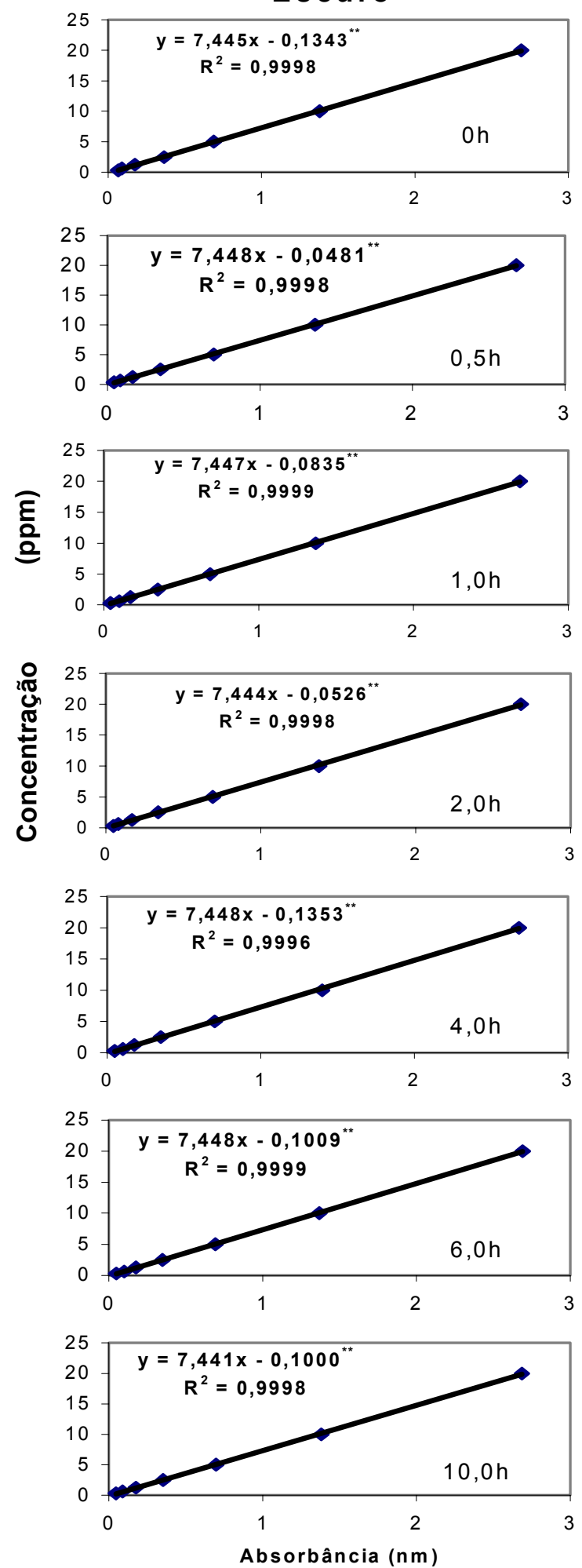

\section{Pleno sol}
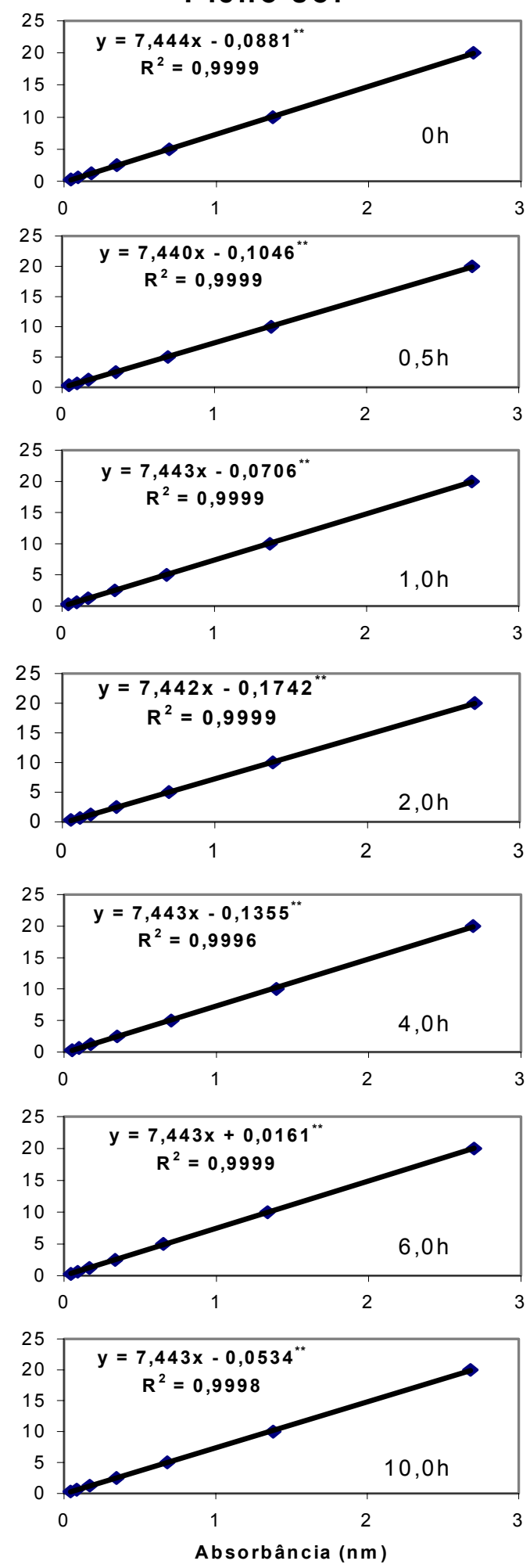

** - Significativo a $1 \%$ de probabilidade.

Figura 1 - Curvas de regressão linear das soluções do corante Azul Brilhante submetido a diferentes períodos de exposição ou não à luz solar. Botucatu-SP, 2003. 


\section{Escuro}
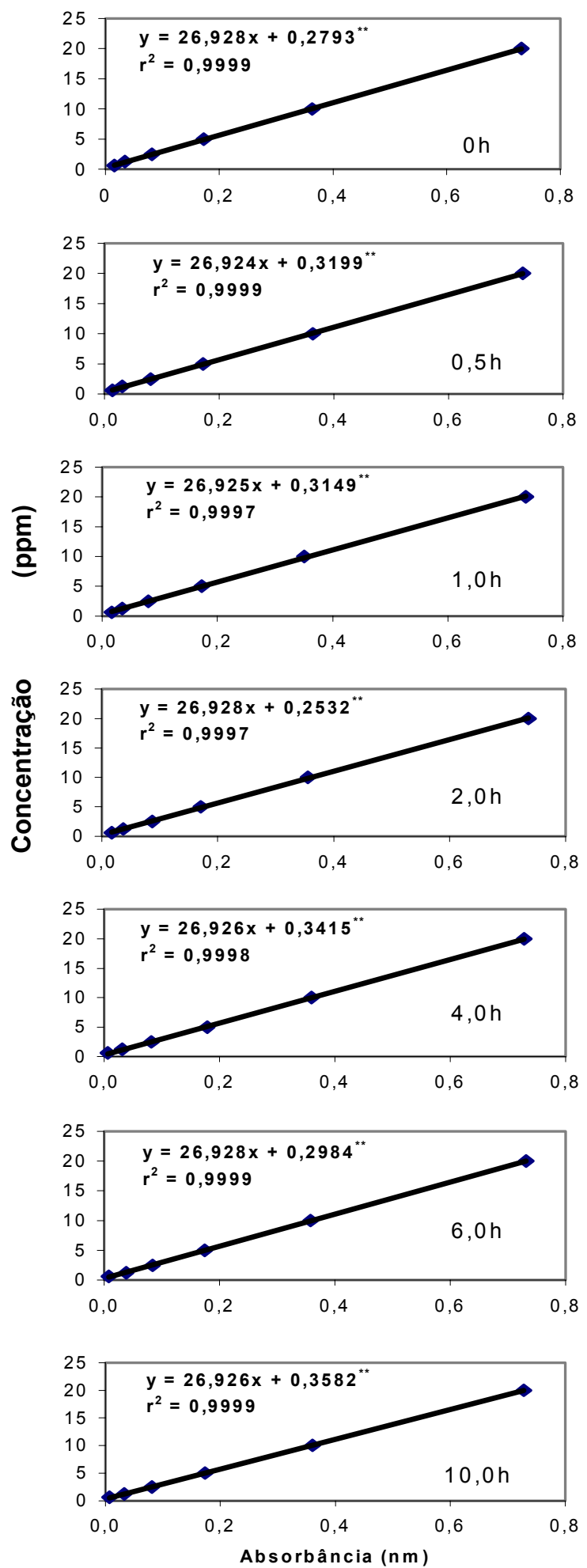

** - Significativo a $1 \%$ de probabilidade.

\section{Pleno sol}
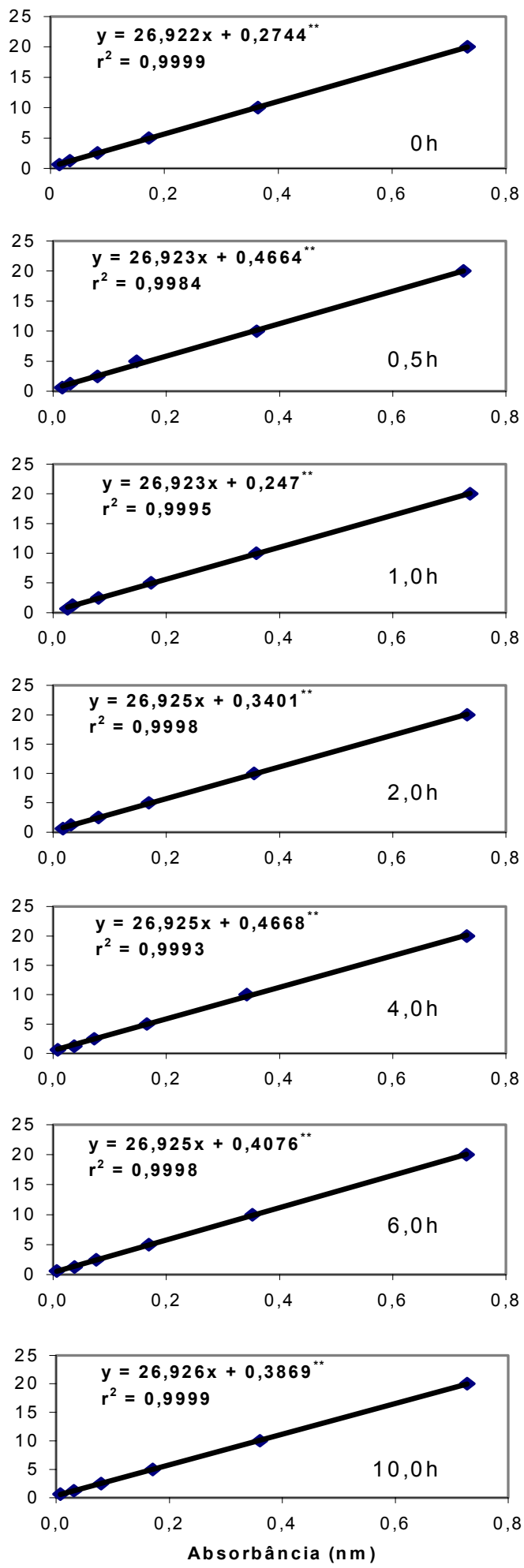

Figura 2 - Curvas de regressão linear das soluções do corante Amarelo Tartrasina submetido a diferentes períodos de exposição ou não à luz solar. Botucatu-SP, 2003. 
diferentes períodos de exposição ou não à luz solar. Pode-se notar na Tabela 1 que as concentrações das soluções (em ppm) de ambos os corantes foram estatisticamente semelhantes entre si, independentemente do tempo de exposição e da condição de luminosidade a que foram submetidas. Cook \& Hislop (1993) estabeleceram que um traçador ideal para os estudos quantitativos de deposição de gotas é aquele que pode ser totalmente extraído ou recuperado no alvo, mesmo se encontrando em estado seco. Os resultados obtidos neste segundo estudo sugerem que os corantes Azul Brilhante e Amarelo Tartrasina também podem ser considerados como traçadores ideais quando utilizados em materiais inertes e mantidos em ambientes abertos durante um período de até 10,0 horas.

O corante Amarelo Tartrasina também mostrou alta estabilidade quando depositado na superficie das folhas do aguapé e submetido a diferentes períodos de exposição ou não à luz solar no terceiro estudo. Na Tabela 2 estão apresentadas as concentrações médias (em ppm) das soluções obtidas após a aplicação dos diferentes tratamentos. Pode-se observar que não ocorreram diferenças estatísticas entre as diversas situações, indicando que o corante Amarelo Tartrasina não apresentou nenhum tipo de interação num intervalo de até $10,0 \mathrm{~h}$ de contato com o tecido foliar e, por isso, pôde ser totalmente extraído da superfície das folhas.

Ainda na Tabela 2, é possível observar que o corante Azul Brilhante apresentou-se estável quando utilizado em alvo natural por um período de até $6,0 \mathrm{~h}$ de contato com a superfície foliar do aguapé. No entanto, as concentrações das soluções obtidas após 10,0 h de contato foram estatisticamente inferiores àquelas obtidas nos demais períodos estudados, indicando que provavelmente ocorreram interações entre o corante e os tecidos foliares. Comparando-se com as concentrações das soluções obtidas a $0,0 \mathrm{~h}$, as perdas para as situações de escuro e pleno sol foram de 7,8 e $18,6 \%$, respectivamente.

Embora não tenha ocorrido diferença estatística, a perda na concentração da solução do corante Azul Brilhante sobre as folhas de aguapé mantidas a pleno sol durante 10,0 h foi maior quando comparada com a condição de não-exposição à luz solar pelo mesmo período, representando uma diminuição de aproximadamente $12 \%$. Palladini (2000) comenta que o corante Azul Brilhante mostrou-se altamente estável quando depositado em folhas individuais de citros por um período de até 8,0 horas, sendo este seu limite máximo de estudo. Entretanto, as folhas de citros encontravam-se destacadas da planta e mantidas túrgidas através da imersão de seus

Tabela 1 - Concentrações dos corantes Azul Brilhante e Amarelo Tartrasina observadas na placa de Petri após os períodos de exposição ou não à luz solar. Botucatu-SP, 2003

\begin{tabular}{|c|c|c|c|c|}
\hline \multirow{2}{*}{ Tempo (h) } & \multicolumn{2}{|c|}{ Azul Brilhante } & \multicolumn{2}{c|}{ Amarelo Tartrasina } \\
\cline { 2 - 5 } & Escuro & Sol & Escuro & Sol \\
\hline 0,0 & 3,2573 & 3,2692 & 3,2172 & 3,2032 \\
\hdashline 0,5 & 3,2515 & 3,2807 & 3,2945 & 3,2673 \\
\hline 1,0 & 3,2725 & 3,2393 & 3,1917 & 3,1830 \\
\hline 2,0 & 3,2500 & 3,2652 & 3,2565 & 3,2968 \\
\hline 4,0 & 3,2647 & 3,2860 & 3,3042 & 3,3142 \\
\hline 6,0 & 3,2758 & 3,2673 & 3,2820 & 3,2685 \\
\hline 10,0 & 3,2592 & 3,2250 & 3,3100 & 3,2868 \\
\hline F hora $(\mathrm{H})$ & \multicolumn{2}{|c|}{$0,24^{\text {ns }}$} & \multicolumn{2}{|c|}{$0,70^{\text {ns }}$} \\
\hline F luz $(\mathrm{L})$ & \multicolumn{2}{|c|}{$0,00^{\text {ns }}$} & \multicolumn{2}{|c|}{$0,02^{\text {ns }}$} \\
\hline F HxL & \multicolumn{2}{|c|}{$0,32^{\text {ns }}$} & \multicolumn{2}{|c|}{$0,05^{\text {ns }}$} \\
\hline CV $(\%)$ & \multicolumn{2}{|c|}{2,4} & \multicolumn{2}{c|}{5,8} \\
\hline d.m.s. & \multicolumn{2}{|c|}{2,4} & 0,2178 \\
\hline
\end{tabular}

n.s. não-significativo.

Tabela 2 - Concentrações dos corantes Azul Brilhante e Amarelo Tartrasina observadas nas folhas de E. crassipes após os períodos de exposição ou não à luz solar. Botucatu-SP, 2003

\begin{tabular}{|c|c|c|c|c|}
\hline \multirow{2}{*}{ Tempo (h) } & \multicolumn{2}{|c|}{ Azul Brilhante } & \multicolumn{2}{c|}{ Amarelo Tartrasina } \\
\cline { 2 - 5 } & Escuro & Sol & Escuro & Sol \\
\hline 0,0 & $3,2200 \mathrm{a}$ & $3,2325 \mathrm{a}$ & 3,2650 & 3,2775 \\
\hline 0,5 & $3,2550 \mathrm{a}$ & $3,2350 \mathrm{a}$ & 3,2425 & 3,2650 \\
\hline 1,0 & $3,2400 \mathrm{a}$ & $3,2225 \mathrm{a}$ & 3,2650 & 3,2575 \\
\hline 2,0 & $3,2950 \mathrm{a}$ & $3,2500 \mathrm{a}$ & 3,2400 & 3,2575 \\
\hline 4,0 & $3,2475 \mathrm{a}$ & $3,2325 \mathrm{a}$ & 3,2525 & 3,2750 \\
\hline 6,0 & $3,2325 \mathrm{a}$ & $3,2325 \mathrm{a}$ & 3,2625 & 3,2900 \\
\hline 10,0 & $2,9950 \mathrm{~b}$ & $2,6325 \mathrm{~b}$ & 3,2700 & 3,2850 \\
\hline F hora $(\mathrm{H})$ & \multicolumn{2}{|c|}{$11,71^{* *}$} & \multicolumn{2}{|c|}{$1,13^{\text {ns }}$} \\
\hline F luz (L) & \multicolumn{2}{|c|}{$3,18^{\text {ns }}$} & \multicolumn{2}{|c|}{$5,61^{\text {ns }}$} \\
\hline F HxL & \multicolumn{2}{|c|}{$1,96^{\text {ns }}$} & \multicolumn{2}{|c|}{$0,37^{\text {ns }}$} \\
\hline CV (\%) & \multicolumn{2}{|c|}{4,2} & \multicolumn{2}{c|}{0,9} \\
\hline d.m.s. & \multicolumn{2}{|c|}{0,1914} & 0,0403 \\
\hline
\end{tabular}

n.s. não-significativo; ** significativo a $1 \%$ de probabilidade; Médias seguidas de mesma letra dentro da coluna não diferem estatisticamente entre si pelo teste $t(p>0,05)$.

Planta Daninha, Viçosa-MG, v. 23, n. 2, p. 287-294, 2005 
respectivos pedúnculos em água destilada. As perdas observadas neste estudo provavelmente estão associadas com a maior atividade fisiológica das folhas integras de aguapé que foram mantidas a plena luz solar, o que poderia ter favorecido possíveis interações entre o corante e o alvo natural e, conseqüentemente, aumentado o porcentual de retenção do corante nos tecidos foliares do aguapé.

Os resultados demonstram que os corantes Azul Brilhante e Amarelo Tartrasina podem ser utilizados em estudos de tecnologia de aplicação com gotas pulverizadas sobre alvos artificiais inertes e mantidos em campo por um período de até 10,0 horas de exposição solar sem que ocorram perdas significativas. O corante Amarelo Tartrasina também pode ser usado em alvos naturais sob as mesmas condições de campo e durante o mesmo período de contato com a superficie foliar. Entretanto, o corante Azul Brilhante apresenta a restrição de contato com alvos naturais integros de no máximo 6,0 horas para que ocorram perdas significativas por retenção nos tecidos foliares de aguapé. Cabe salientar que a extração de ambos os corantes pode variar em função das características morfológicas da folha e da espécie-alvo a ser utilizada nos estudos quantitativos de deposição de gotas pulverizadas sobre alvos naturais íntegros.

\section{LITERATURA CITADA}

COOK, B. K.; HISLOP, E. C. Spray tracing techniques. In: MATTHEWS, G. A.; HISLOP, E. C. (Eds.) Application technology for crop protection. Wallinford: CAB, 1993. p. $85-100$.

COSTA, N. V. et al. Deposição de gotas de pulverização e $\mathrm{pH}$ foliar em plantas daninhas aquáticas. In: CONGRESSO BRASILEIRO DA CIÊNCIA DAS PLANTAS

DANINHAS, 24., 2004, São Pedro. Resumos... São Pedro: SBCPD, 2004. CD-ROM.

HAYDEN, J. et al. Two water-soluble optically resolvable dyes for comparing pesticide spray distribution. J. Econ. Entomol., v. 83, n. 6, p. 2411-2413, 1990.
MATUO, T. Desenvolvimento de um pulverizador intermitente operado fotoeletricamente para tratamento de pomares de citrus. $1988.167 \mathrm{f}$. Tese (Livre Docência) - Universidade Estadual Paulista, Jaboticabal, 1988.

NEGRISOLI, E. et al. Depósitos unitários de calda de pulverização com e sem surfatante em plantas de Salvinia molesta. Planta Daninha, v. 20, p. 51-56, 2002. (Edição especial)

PALLADINI, L. A. Metodologia para avaliação da deposição em pulverizações. 2000. $111 \mathrm{f}$. Tese (Doutorado em Proteção de Plantas) - Universidade Estadual Paulista, Botucatu, 2000.

PERGHER, G.; GUBIANI, R.; TONETTO, G. Foliar deposition and pesticide losses from three air-assisted sprayers in a hedgerow vineyard. Crop Protec., v. 16, n. 1, p. 25-33, 1997.

SILVA, J. R. V.; MARTINS, D.; DOMINGOS, V. D. Deposição da calda de pulverização em dois estágios de desenvolvimento de plantas de Typha subulata. In: CONGRESSO BRASILEIRO DA CIÊNCIA DAS PLANTAS DANINHAS, 24., 2004, São Pedro. Resumos... São Pedro: SBCPD, 2004. CD-ROM.

SOUZA, R. T.; MACIEL, C. D. G.; VELINI, E. D. Avaliação dos depósitos unitários de calda de pulverização em plantas daninhas da cultura da soja. In: CONGRESSO BRASILEIRO DA CIÊNCIA DAS PLANTAS DANINHAS, 22., 2000, Foz do Iguaçu. Resumos... Foz do Iguaçu: SBCPD, 2000. p. 473.

TERRA, M. A. et al. Efeito de diferentes concentrações de Aterbane na deposição de calda em plantas de alface d'água (Pistia stratiotes). In: CONGRESSO BRASILEIRO DA CIÊNCIA DAS PLANTAS DANINHAS, 24., 2004, São Pedro. Resumos... São Pedro: SBCPD, 2004. CD-ROM.

TOFOLI, G. R. Efeito do tamanho do alvo e condições operacionais sobre a uniformidade de deposição de pulverizações em pré-emergência. 2001. $62 \mathrm{f}$. Dissertação (Mestrado em Proteção de Plantas) Universidade Estadual Paulista, Botucatu, 2001.

YATES, W. E.; AKESSON, N. B. Fluorescent tracers for quantitative microresidue analysis. Am. Soc. Agric. Eng., v. 6, p. 105-114, 1963. 